\title{
Electric polarization effects on the electronic spectral shift of centrosymmetric compounds
}

\author{
Denisio M. Togashi ${ }^{\mathrm{a}, *}$, Sílvia M.B. Costa ${ }^{\mathrm{a}}$, Abílio J.F.N. Sobral ${ }^{\mathrm{b}}$, \\ A.M. d'A.R. Gonsalves ${ }^{b}$ \\ ${ }^{\text {a }}$ Centro de Química Estrutural, Complexo I - Instituto Superior Técnico, 1049-001 Lisbon, Portugal \\ ${ }^{\mathrm{b}}$ Departamento de Química, Universidade de Coimbra, P-3000 Coimbra, Portugal
}

Received 19 January 2004; accepted 18 February 2004

\begin{abstract}
The classic dielectric dipolar Onsager model was extended to include quadrupolar interactions between solute molecules and solvents with different polarities. A multiparametric solvatochromic expression, based on the point quadrupole moment inside a spherical cavity embedded in a dielectric continuum, is applied to centrosymmetric sulfonamide porphyrins, zinc tetraphenyl porphyrin, squaraine and 9,10-dicyanoanthracene, in order to account for the quadrupolar polarization effect of solute molecules. The reaction field polarity functions created respectively by dipole and quadrupole moments are compared and found to be linearly correlated.
\end{abstract}

(C) 2004 Elsevier B.V. All rights reserved.

Keywords: Quadrupole moment; Reaction field; Polarization; Dielectric; Solvatochromism

\section{Introduction}

The solvent effect on the electronic spectra has been the subject of many investigations [1-5]. A decrease in the transition energies in solution $(v)$ as compared to vacuum $\left(v_{0}\right)$ is generally found. This is basically due to induced dipoles associated to the polarizability of solute and solvent molecules. It is also due to the dipole transition moment of solute molecules interacting with the polarizability of solvent molecules. Therefore, in general, the spectral shift is correlated with the solvent refraction index function $f\left(n^{2}\right)=\left(n^{2}-1\right) /\left(2 n^{2}+1\right)$, which takes into account such solvent interactions.

The dipolar interactions involving solute-solvent molecules were taken into account in Onsager's reaction field model where the solute polar molecule is represented by a point dipole at the center of a sphere [6]. Subsequently, using concepts of Onsager reaction field, the perturbation quantum theory treatment of such dipolar interactions was developed and improved by

\footnotetext{
${ }^{*}$ Corresponding author. Fax: +351-21-846-4455.

E-mail address: togashi@ist.utl.pt (D.M. Togashi).
}

Bayliss, McRae and Ooshika (BMO) [1,7-9]. Thus, the electronic spectral shift of a molecular solute, in the absence of specific interactions, is described by the following equation:

$$
\begin{aligned}
v= & v_{0}+(A+B+C) \times f\left(n^{2}\right)+E \times f\left(\varepsilon, n^{2}\right) \\
& +F \times f\left(\varepsilon, n^{2}\right)^{2},
\end{aligned}
$$

where $f\left(\varepsilon, n^{2}\right)=(\varepsilon-1) /(\varepsilon+2)-\left(n^{2}-1\right) /\left(n^{2}+2\right)$, is known as the orientation polarization function and its quadratic term accounts for the Stark effect. The terms $A, B, C, E$ and $F$ are functions of the solute property at ground and excited states [1-4].

The electronic spectra of centrosymmetric molecules like porphyrins are normally correlated with the $f\left(n^{2}\right)$ function which means that induced polarization and dispersion forces are mainly responsible for the spectral shift observed in these molecules [10]. However, if intramolecular and/or intermolecular (solute-solvent) non-covalent interactions are present in the porphyrin derivatives, certain conformations can have different spectroscopic behavior leading to significant spectral shifts caused by the medium polarity. This possibility has already been studied by equilibria between 
conformers with either zero or non-zero dipole moments [11-13].

It has been reported that the dielectric dipolar BMO model is the best one at expressing the solvatochromism of centrosymmetric molecules, which do not have dipole moment [14-17]. The possibility that the correlation formed therein was due to the solute quadrupole moment and the solvent permanent dipole moment interactions was previously advanced $[15,16]$. Theoretical investigations, like quantum mechanics studies $[3,18,19]$ or molecular mechanic calculations [20], have extended the Onsager concept to a multipole in a spherical cavity, not only to dipole, but quadrupole, octupole, and so on. Thus, it is likely that some correlation would be observed in the dipolar BMO model for the cases where the quadrupole moment might be significant. Nevertheless, the solvatochromic shift of centrosymmetric molecules has not yet become a standard tool and only few cases have been reported [14-17,21,22].

Suppan and Ghoneim $[5,22]$ have proposed an expression that takes into account the quadrupolar and dipolar interactions between the solvent and solute molecules as shown in the following equation:

$v=v_{0}-\frac{\left(\Theta_{\mathrm{ex}}^{2}-\Theta_{\mathrm{gr}}^{2}\right)}{a^{5}} f\left(n^{2}\right)-\frac{\Theta_{\mathrm{gr}}\left(\Theta_{\mathrm{ex}}-\Theta_{\mathrm{gr}}\right)}{2 a^{5}} f\left(\varepsilon, n^{2}\right)$.

These authors used the averaged quadrupole-dipole potential to obtain the solvation energy of the solute quadrupole moment on the ground $\left(\Theta_{\mathrm{gr}}\right)$ and on the excited states $\left(\Theta_{\mathrm{ex}}\right)$ at a distance $a$ (Onsager cavity radius) from the solvent dipole moment. The development of the orientation polarization term, $f\left(\varepsilon, n^{2}\right)$, in the reaction field was obtained by the comparison of Debye and Onsager functions already built for the spherical and dipolar solute molecules. Therefore, expression (2) treats specifically the solute quadrupole and solvent dipole averaged interactions but using the dipolar solvent reaction field.
In this paper, we present a dielectric model which takes also into account the interaction of the solute quadrupole moment with the solvent using the classical electrostatic Onsager theory, and the literature approach for the polarization effects on the non-equilibrium situation [19,23-25]. In other words, the main goal is to improve the Suppan and Ghoneim expression setting on the correct reaction field developed by the quadrupole moment applicable to non-dipolar solute molecules. Thereafter, the quadrupolar and the dipolar dielectric models are compared to understand how the BMO model can interpret the spectroscopic properties of centrosymmetric molecules with non-dipole moment. The model is used to analyse solvatochromic effects detected in the electronic transitions of centrosymmetric molecules such as two different sulfoamido (with or without aliphatic chains) meso-tetraphenylporphyrin derivatives (see Scheme 1) whereas meso-tetraphenyl porphyrin (TPP) was used as the reference molecule, and three other centrosymmetrical molecules (zinc tetraphenylporphyrin, squaraine and 9,10-dicyanoanthracene) for which the experimental data in solvents with different polarities is available in the literature $[14,15,26]$.

\section{Quadrupole dielectric model}

The quadrupole moment is better described as a set of electrical charges which form two equal and opposite dipoles. For an axial quadrupole moment these dipole moments are along the same symmetrical axis [22,27]. The general description quadrupole moment tensor is

$\mathbf{Q}=\frac{1}{2} \sum_{i} e_{i}\left(3 r_{i} \cdot r_{i}-r_{i}^{2} I\right)$,

where $e_{i}$ is the electric charge at distance $r_{i}, I$ is the unit tensor and $r_{i}^{2}=x_{i}^{2}+y_{i}^{2}+z_{i}^{2}$. The scalar quantity or

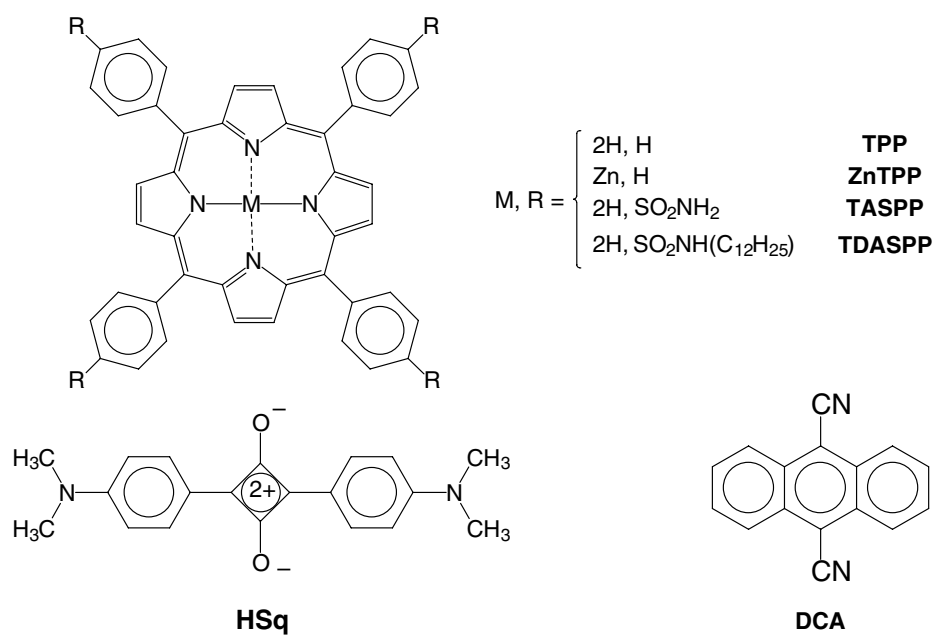

Scheme 1. Molecular structures of centrosymmetric compounds. 
quadrupole strength of $\mathbf{Q}$ depends on the choice of the coordinate's origin. However, if one of the axis ( $z$-axis for instance) is taken along the axis of the quadrupole moment, the non-zero components are related as follows:

$\Theta=Q_{z z}=-2 Q_{x x}=-2 Q_{y y}$,

where $\Theta$ is often used as the scalar quadrupole strength of the quadrupole moment tensor $\mathbf{Q}$ [27].

In order to include the quadrupolar interaction in solvatochromic studies using the Onsager model, it is necessary to find the reaction field caused by the solvent polarization induced by the potential field of the quadrupole moment of the solute molecule inside the spherical cavity of radius $a$. Therefore, developing the Onsager model for an axial quadrupole moment (see Appendix A) the reaction field is given by

$R^{(Q)}=Q(\varepsilon) \frac{\Theta}{a^{5}}$,

where

$Q(\varepsilon)=\frac{3(\varepsilon-1)}{3 \varepsilon+2}$.

The same reaction field (Eq. (5)) has already been used in previous studies [11-13] to investigate the equilibrium solvation of solute molecules on the ground state. However, a non-equilibrium situation is created when excited species are involved. In such cases, the solvent polarization by the solute molecule is normally divided in two components [19,23-25]. A fast component or induced polarization is due to the fast movement of the solvent molecule electronic density that is in rapid equilibrium with the solute charge distribution on its electronic excitation. The second component, orientation polarization, is much slower and represents the solvent molecules movement upon the excitation of solute molecules. The electronic polarization of solvent molecules is induced and the equilibrium charge distribution of the solute molecules in the excited state is quickly achieved. By contrast, the orientation polarization of solvent molecules results from the charge distribution of solute molecules in the ground state. The electronic component of the reaction field appears when the solvent molecules nuclei cannot follow the changes in the external field (excitation) or in the dielectric constant at high-frequency fields. Using the Maxwell relation for the dielectric constant and the refractive index, $\varepsilon=n^{2}$, the reaction field for induced polarization for the quadrupole moment is

$R_{\text {ind,ex }}^{(Q)}=Q\left(n^{2}\right) \frac{\Theta_{\mathrm{ex}}}{a^{5}}$.

Consequently, the orientation polarization reaction field is the difference between the total reaction field and the induced polarization

$R_{\mathrm{or}, \mathrm{gr}}^{(Q)}=Q\left(\varepsilon, n^{2}\right) \frac{\Theta_{\mathrm{gr}}}{a^{5}}$, where

$Q\left(\varepsilon, n^{2}\right)=Q(\varepsilon)-Q\left(n^{2}\right)$.

Recently, the separation of the reaction field polarization has been reviewed due to some mixing degree of both components [23]. Nevertheless, if the final free energy terms for the spectral shifts are not influenced such mixing can be ignored. Therefore, in accordance with the reported treatment [28], the free energy of the excited state immersed in the solvent molecules, represented by the quadrupole moment inside the spherical cavity polarizing the continuum dielectric, is

$$
\begin{aligned}
\Delta G_{\mathrm{ex}}= & -\frac{1}{2} R_{\mathrm{gr}}^{(Q)} \cdot \Theta_{\mathrm{gr}}-\int_{0}^{1}\left(\Theta_{\mathrm{ex}}-\Theta_{\mathrm{gr}}\right) \\
& \times\left[R_{\mathrm{gr}}^{(Q)}+Q\left(n^{2}\right) \cdot \xi \frac{\left(\Theta_{\mathrm{ex}}-\Theta_{\mathrm{gr}}\right)}{a^{5}}\right] \mathrm{d} \xi .
\end{aligned}
$$

After rearrangement,

$\Delta G_{\mathrm{ex}}=-\frac{1}{2} Q(\varepsilon) \frac{\Theta_{\mathrm{ex}}^{2}}{a^{5}}+\frac{1}{2} Q\left(\varepsilon, n^{2}\right) \frac{\left(\Theta_{\mathrm{ex}}-\Theta_{\mathrm{gr}}\right)^{2}}{a^{5}}$.

The free energy of the solute molecules in the ground state is known, once the system is in equilibrium:

$\Delta G_{\mathrm{gr}}=-\frac{1}{2} Q(\varepsilon) \frac{\Theta_{\mathrm{gr}}^{2}}{a^{5}}$.

Thus, the spectral shift on the electronic absorption is

$v=v_{0}+\Delta G_{\text {ex }}-\Delta G_{\mathrm{gr}}$.

Substituting Eqs. (11) and (12) into Eq. (13) and rearranging it, finally gives

$v=v_{0}-\frac{\left(\Theta_{\mathrm{ex}}^{2}-\Theta_{\mathrm{gr}}^{2}\right)}{2 a^{5}} Q\left(n^{2}\right)-\frac{\Theta_{\mathrm{gr}}\left(\Theta_{\mathrm{ex}}-\Theta_{\mathrm{gr}}\right)}{a^{5}} Q\left(\varepsilon, n^{2}\right)$.

The development of a quadrupole interaction based on the Onsager theory does not describe all the possible interactions which arise on the centrosymmetric molecule solvatochromism. Also it is assumed that the molecular quadrupole moment can be reduced to an axial symmetry representation. Furthermore, there are two main points which must be noted with this methodology: (i) the effect of the reaction field of quadrupole moment on the polarizability of the solute molecule was not taken into account, and (ii) it is assumed that the centrosymmetric molecule is in a spherical cavity. It is clear that the reaction field created by the centrosymmetric solute quadrupole moment will affect the coefficients of its quadrupole tensor due to the molecular polarizability. However, effects which depend on the induced quadrupole moment of solute molecule (Stark effect) were neglected here. In spite of these limitations, there are other solvent-solute interactions that have to be included in the general spectral shifts such as: (a) the solute polarizability and permanent dipole moment from solvent molecules, (b) the dispersion forces and (c) 


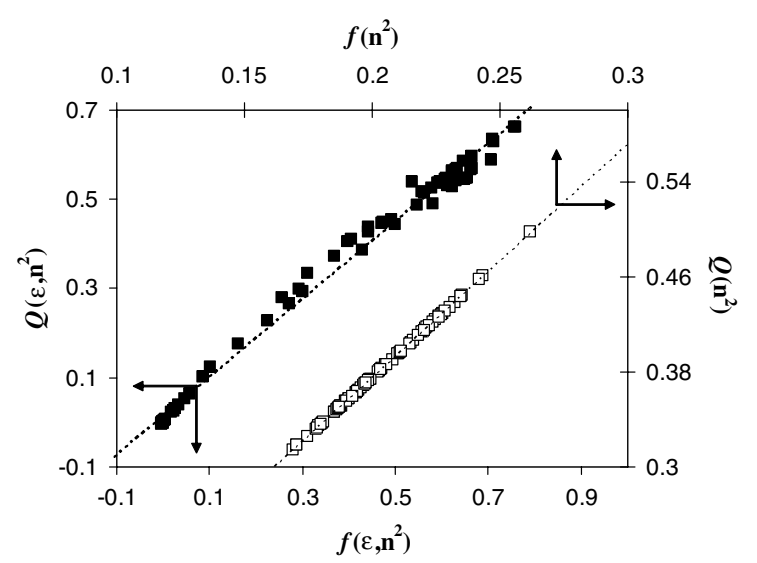

Fig. 1. Linear correlations between the solvent reaction field factors (without the cavity radii) for dipole and quadrupole moments. Open symbols correspond to induced polarization and close symbols to orientation polarization (BMO description for dipole case).

the solvent interaction with the transition dipole moment created during the excitation. All these effects are known to be proportional to the solvent $f\left(n^{2}\right)$ function. It is interesting to note that for a large number of solvents $[29,30]$, the $f\left(n^{2}\right)$ is proportional to the $Q\left(n^{2}\right)$ (Fig. 1). Therefore, the final expression for the spectral shifts taking into account the quadrupolar interaction is $v=v_{0}-\Pi \cdot f\left(n^{2}\right)-\Xi \cdot Q\left(\varepsilon, n^{2}\right)$,

where the $\Pi$ parameter represents the interactions which involve the solute and/or solvent molecular polarizabilities like induced polarization and dispersion forces. $\Xi$ is proportional to the product between the ground state quadrupole moment and the difference between excited and ground state quadrupole moments, i.e. $\Xi=\Theta_{\mathrm{gr}}\left(\Theta_{\mathrm{ex}}-\Theta_{\mathrm{gr}}\right) / a^{5}$.

In our model, these interactions are represented by the solute quadrupole/dielectric solvent built in the solvent reaction field caused by the quadrupolar solute polarization. The Suppan and Ghoneim approach works because a good linearity is observed between $f\left(\varepsilon, n^{2}\right)$ and $Q\left(\varepsilon, n^{2}\right)$ (Fig. 1). This linearity explains the good results obtained for other centrosymmetric molecules to which the BMO model was applied to study solvatochromic effects [14-17].

\section{Experimental}

\subsection{Materials}

The porphyrin derivatives were synthesised and already described [31]. TPP was purchased from SigmaAldrich and used as received. $n$-Butanol, n-pentanol, $n$-decanol, 1,3-propanediol, 1,4-propanediol, 1,5-pentanediol and propylene carbonate were analytical grade, all other solvents were spectroscopic grade and were used without any further treatment. The sample concentrations were approximately $(1-3) \times 10^{-6} \mathrm{M}$.

\subsection{Apparatus}

The electronic absorption spectra were recorded with a JASCO V560 UV/Vis spectrophotometer with blank correction and fluorescence spectra with a Perkin-Elmer LS-50B spectrofluorimeter. The instrumental response at each wavelength was corrected by means of a curve provided with the instrument. The sample holder of both instruments was thermostated at $25^{\circ} \mathrm{C}$ and all the measurements were made using quartz cuvette $10 \mathrm{~mm}$ path length. The refraction index $(n)$ and the dielectric constant $(\varepsilon)$ are literature values [29,30]. The linear and multilinear correlations were performed using Microsoft Excel $^{\circledR}$ and the statistical significance of the parameters were analysed by $t$ statistic values, single tail, with the respective degrees of freedom and 95\% of confidence [29]. The spectral shifts maxima for each parameter (the parameter weight) were calculated multiplying a parameter coefficient (obtained from the correlations) by the range of this parameter in the solvents studied [32].

\section{Results and discussion}

\subsection{Electronic transitions in Porphyrins}

The electronic absorption and fluorescence spectra observed for TDASPP are shown in Fig. 2(a), (b) and appropriate data for the porphyrins studied are presented in Table 1. The spectral shifts observed for the porphyrins were studied using the solvatochromic models. Although the fluorescence maxima $(0,0)$ were obtained, the range of spectral shifts is small (as shown for TDASPP in Fig. 2(b)) reducing the analysis accuracy and lead to values with considerable uncertainty for the models applied.

The shape of the absorption and fluorescence bands in the porphyrins (Scheme 1) are independent of substituent groups and the solvent polarity, except in solvents where the porphyrins' solubility is low. The band profiles are broad indicating the presence of aggregates like that observed for TDASSP in 1,2-ethanediol and 1,3-propanediol. These solvents were discarded from the correlations.

The substituent effect on the electronic transition without the solvent effect can be observed on $v_{0}$ values obtained for the electronic transition in vacuum. The values obtained from the correlation with any model show that $v_{0}$ value of the TPP Soret band is close to the experimental one $\left(25,114 \mathrm{~cm}^{-1}\right)$ [33].

In general, electron-donating substituents at para position lead to red shifts for all the absorption bands in TPP [34]. The red shift is observed for the $v_{0}$ value for 

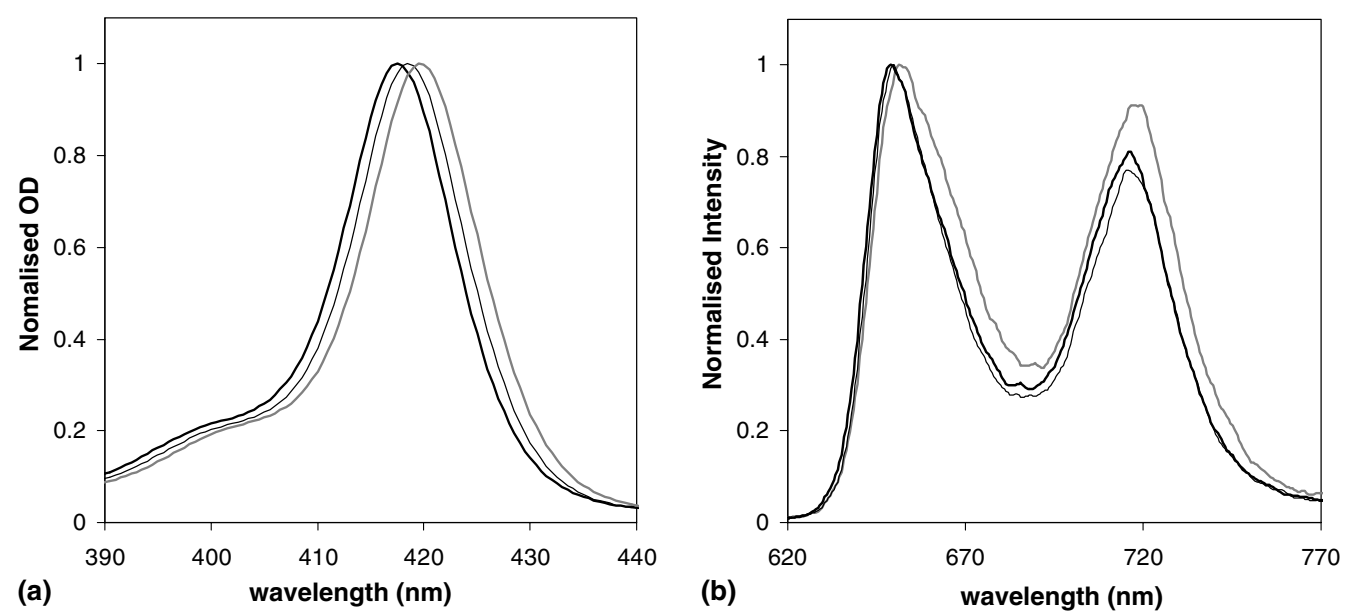

Fig. 2. Electronic spectra of TDASPP in chloroform (grey line), $N, N$-dimethylformamide (straight line) and propylene carbonate (bold line). (a) Soret absorption band region and (b) fluorescence emission.

the TPP Soret band (Tables 2 and 3), but aminosulfonyl group is considered an electro-withdrawing substituent [30]. The red shifts were also reported for other electronwithdrawing substituents at para position on TPP like methylsulfonyl, cyano, chloro and bromo [34]. In the same work, it was proposed that the spectral shifts of

Table 1

Porphyrin wavelengths $(\mathrm{nm})$ for Soret band absorption

\begin{tabular}{llll}
\hline Solvent & TPP & TASPP & TDASPP \\
\hline Methanol & 412 & 415 & 415 \\
Ethanol & 413 & 416 & 416 \\
$n$-Propanol & 414 & 417.5 & 417 \\
$n$-Butanol & 414.5 & 418.5 & 417.5 \\
$n$-Petanol & 415 & 419 & 417.5 \\
$n$-Hexanol & 415.5 & 419.5 & 418 \\
$n$-Octanol & 415.5 & 420 & 418 \\
$n$-Decanol & 416 & 421.5 & 419 \\
1,2-Ethanediol & 415.5 & 418.5 & 424 \\
1,3-Propanediol & 416 & 419 & 422 \\
1,4-Butanediol & 416.5 & 420 & 419 \\
1,5-Pentanediol & 416.5 & 420 & 419 \\
Tetrahydrofuran & 415 & 418.5 & 418 \\
Chloroform & 417.5 & - & 419.5 \\
Acetone & 413 & 416.5 & 416 \\
$N, N$-Dimethylformamide & 416 & 419.5 & 418.5 \\
Dimethylsulfoxide & 417.5 & 420.5 & 420 \\
Acetonitrile & 412.5 & 416 & 415.5 \\
Propylene carbonate & 415 & 418 & 417.5 \\
\hline
\end{tabular}

TPP are better described by a mixture of resonance and inductive effects using Hammett correlations. Alternatively, the molecular polarizability, that is proportional to molecular volume, correlates with the highest occupied molecular orbital (HOMO) and lowest unoccupied molecular orbital (LUMO) energy separation. When the molecular polarizability is large, the electronic distribution may be freely distorted and this is better achieved with small HOMO-LUMO gap energy. Since the presence of substituent groups in the TPP causes an increase of polarizability, the HOMO-LUMO energy separation is reduced and then red shifts on the electronic transitions are expected. In fact, the electronic differences on different substituent groups will impinge on the band already red shifted.

\subsection{Spectral shifts correlated by dipolar dielectric model}

It is known that $N$-methylmethane sulfonamide has a dipole moment of 6.2 Debye [35] and the long alkyl groups in $N$-( $p$-tolyl)dodecyl sulfonamide can interact amongst themselves [36]. Therefore it would be possible that the $N$-dodecylaminosulfonyl substituent group in TDASPP could induce a dipolar conformer.

The spectral shifts data in Table 2 for TPP and sulfonamide derivatives are well correlated with the pa-

Table 2

Correlation of porphyrin Soret bands by BMO model

\begin{tabular}{|c|c|c|c|c|c|c|}
\hline Compound & $v_{0}\left(\mathrm{~cm}^{-1}\right)$ & $A+B+C\left(\mathrm{~cm}^{-1}\right)$ & $E\left(\mathrm{~cm}^{-1}\right)$ & $F\left(\mathrm{~cm}^{-1}\right)$ & $r$ & $n$ \\
\hline ТPP & $25103 \pm 72$ & $-6332 \pm 237$ & $855 \pm 235$ & $-715 \pm 225$ & 0.992 & 19 \\
\hline TASPP & $24979 \pm 347$ & $-5836 \pm 559$ & $-192 \pm 1307$ & $490 \pm 1144$ & 0.966 & 18 \\
\hline TDASPP & $24893 \pm 60$ & $-5395 \pm 278$ & $344 \pm 261$ & $-247 \pm 251$ & 0.988 & 17 \\
\hline $\mathrm{ZnTPP}$ & $25669 \pm 172$ & $-8343 \pm 869$ & $-1385 \pm 273$ & $1078 \pm 419$ & 0.981 & 19 \\
\hline $\mathrm{HSq}$ & $17985 \pm 298$ & $-8676 \pm 1340$ & $-309 \pm 377$ & $-820 \pm 493$ & 0.902 & 25 \\
\hline HSq* & $17524 \pm 342$ & $-8316 \pm 1540$ & $-203 \pm 434$ & $-1311 \pm 567$ & 0.912 & 25 \\
\hline DCA & $25877 \pm 564$ & $-10533 \pm 2546$ & $-65 \pm 402$ & $-579 \pm 760$ & 0.884 & 13 \\
\hline
\end{tabular}


Table 3

Parameters from quadrupole dielectric model (Eq. (15))

\begin{tabular}{llllll}
\hline Compound & $v_{0}\left(\mathrm{~cm}^{-1}\right)$ & $\Pi\left(\mathrm{cm}^{-1}\right)$ & $\Xi\left(\mathrm{cm}^{-1}\right)$ & $r$ & $n$ \\
\hline TPP & $25193 \pm 78$ & $5993 \pm 303$ & $-170 \pm 52$ & 0.987 & 0.965 \\
TASPP & $24662 \pm 160$ & $5395 \pm 566$ & $-551 \pm 130$ & 0.988 & 18 \\
TDASPP & $24900 \pm 72$ & $5200 \pm 289$ & $-131 \pm 49$ & 0.978 & 17 \\
ZnTPP & $25865 \pm 173$ & $9356 \pm 867$ & $2345 \pm 133$ & 0.868 & 0.863 \\
HSq & $18288 \pm 348$ & $9746 \pm 1560$ & $4148 \pm 531$ & 25 \\
HSq & $17934 \pm 428$ & $9719 \pm 1923$ & $1138 \pm 422$ & 0.876 & 13 \\
DCA & $25506 \pm 352$ & $8819 \pm 1539$ & & & 25 \\
\hline
\end{tabular}

rameters from the BMO model. It is also noted that the more important interactions involve the dispersive forces, accounted for by the $A+B+C$ values, which cause the decrease in the transition energy while $E$ and $F$ values which are dependent on the solute dipole moment have a low contribution to the spectral shift. Therefore, the dispersive forces are the most predominant interactions responsible for the general red shift and no relevant dipolar interactions between porphyrins and solvent molecules were observed.

The $A+B+C$ values found for the TPP derivatives, have absolute values which are smaller than those corresponding to TPP (Tables 2 and 3 ). This indicates that, perhaps the presence of "sulfa" groups affects the dispersion forces. These interactions are proportional to the molecular polarizability and inversely proportional to the cubic cavity radius, $a^{3}$. Therefore, the shift will depend on excited and ground states polarizabilities difference, $\Delta \alpha=\alpha_{\mathrm{ex}}-\alpha_{\mathrm{gr}}$, and cavity radii.

The presence of substituent groups will increase the polarizabilities of ground and excited states. The cavity radius will also increase and finally the effect observed will be a balance between the $\Delta \alpha$ and cavity radius. Assuming that only the dispersive forces are present in solute and solvent molecules, the polarizability variations, $\Delta \alpha$, can be calculated using the Lorentz-Lorentz function $\phi\left(n^{2}\right)=$ $\left(n^{2}-1\right) /\left(n^{2}+2\right)[10]$ shown in the following equation:

$v=v_{0}+p \times \phi\left(n^{2}\right)$,

where

$p=-\frac{3}{2} \frac{\Delta \alpha}{h c a^{3}}\left(\frac{I_{\Sigma} \times I_{\sigma}}{I_{\Sigma}+I_{\sigma}}\right)$.

The solute Onsager cavity radius is already defined by $a$, $I_{\Sigma}$ and $I_{\sigma}$ are the ionization potentials of solvent and solute, respectively, $h$ is the Planck constant and $c$ is the speed of light. It is assumed that the ionization potentials for the porphyrins and solvent molecules equal to $(7.2 \pm 0.4)$ and $(10 \pm 1) \mathrm{eV}$, respectively. Such values are the average of ionization potentials for the different tetraarylporphyrins [37] and the solvents used in this work $[29,30]$. The Onsager cavity was obtained from molecular models [38] where the molar volumes were calculated. The $\Delta \alpha$ obtained for TPP, TASPP and TDASPP are (16 \pm 4$),(20 \pm 9)$ and $(40 \pm 9) \AA^{3}$, respec- tively. The positive values mean that for Soret electronic transition, the polarizability of the excited molecule is higher than that of the ground state. The value for TPP is approximately two times lower from the reported one [10] mainly due to the difference of cavity radius approach. Therefore, in spite of radii differences, the effect observed with substituents is caused by an increase of polarizability difference between excited and ground state in the order: TPP $<$ TASPP $<$ TDASPP, that is, the dispersion forces are balanced by the size effect.

\subsection{Spectral shifts correlated by quadrupole dielectric model}

The results obtained using Eq. (15) are presented in Fig. 3(a) and collected in Table 3. Now, the parameters obtained from the bilinear correlations can be compared to each other with regard to the quadrupole moment of the porphyrin. Similarly to the previous results, the general red shift is observed from dispersion interactions represented now by the $\Pi$ parameter. Although these interactions are still important, the $\Pi$ values show that the presence of the polar aminosulfonyl group in the phenyl of TPP, in general, decreases the weight of such interactions by comparison to TPP (see Table 4). The weight of the quadrupolar interaction is still lower than the dispersion interaction. However, if the quadrupolar interactions were more important than the dispersion interactions, a blue shift of the absorption Soret band of TASPP would be expected due to the reduction of the strength of the quadrupole moment, that is, $\Theta_{\mathrm{ex}}<\Theta_{\mathrm{gr}}$ (see the negative values for $\Xi$ parameter in Table 3 ).

It is quite difficult to analyse the effect of chain length on the quadrupole moment, even when comparing with the corresponding value for TPP due to the difference in the assumption of cavity radius [38]. Even so, assuming such values, the contribution of the cavity size on quadrupole interaction, that is $a$ to the fifth power is expected to reduce proportionally the quadrupole interaction from 1, 1.4 and 5.7 for TPP, TASPP and TDASPP, respectively. But, it is seen (from the values of the $\Xi$ parameter in Table 3 ) that the contribution of the quadrupole interaction is higher in TASPP, and lower when one hydrogen atom is substituted by dodecyl groups (TDASPP). In other words, the bulky groups do 

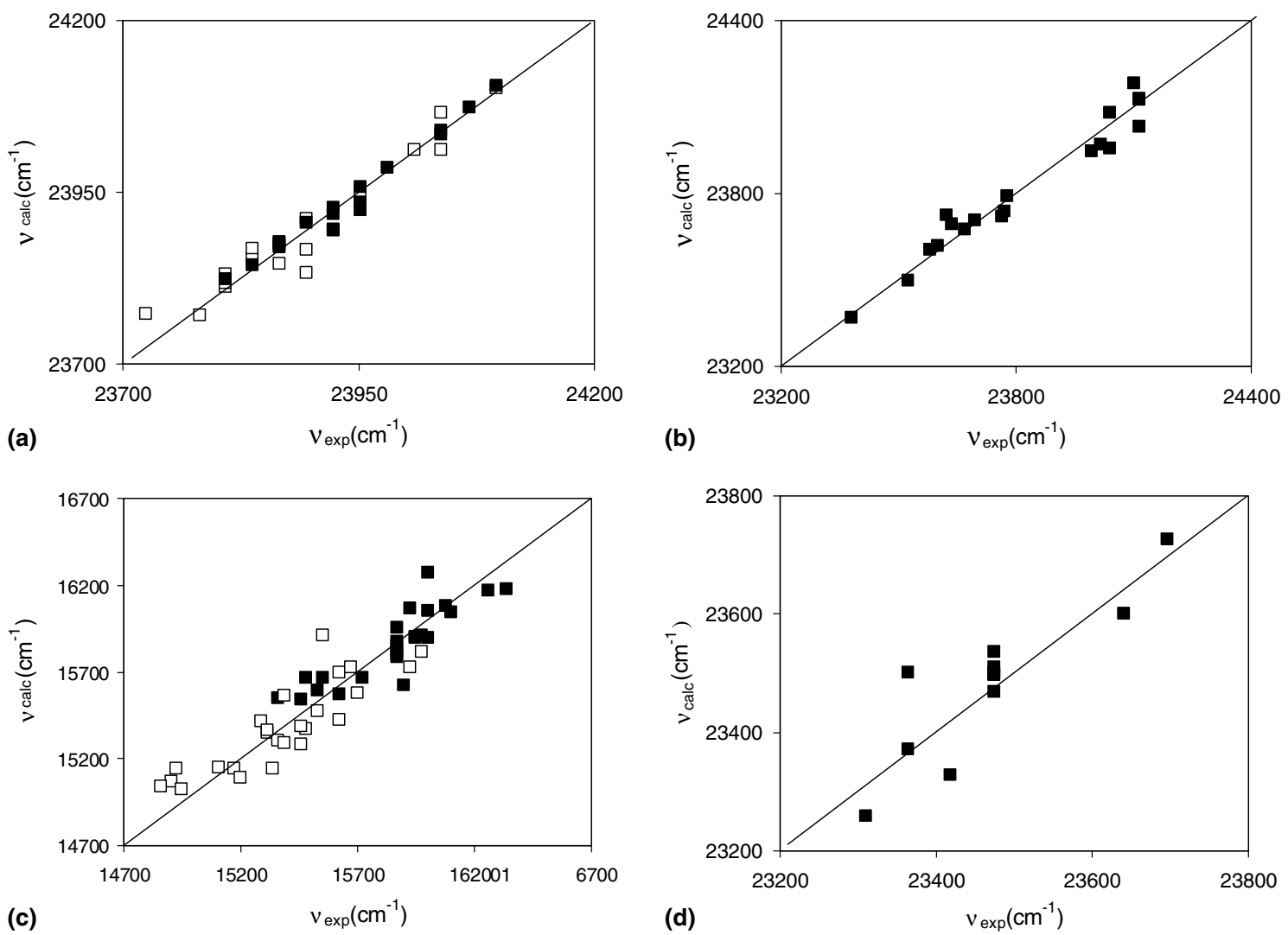

Fig. 3. Multilinear correlations of Eq. (15) with the Soret band shifts of TASPP (open symbols) and TDASPP (close symbols) (a) and with the Soret band shifts of ZnTPP (b). In addition, the correlations with absorption maxima of HSq (closed symbol) and fluorescence maxima of HSq* (opened symbol) in (c) and DCA in (d).

Table 4

Maximum range of variation of parameters from quadrupole dielectric model

\begin{tabular}{llc}
\hline Compound & $\Pi \times \Delta f\left(n^{2}\right)\left(\mathrm{cm}^{-1}\right)$ & $\Xi \times \Delta Q\left(\varepsilon, n^{2}\right)\left(\mathrm{cm}^{-1}\right)$ \\
\hline TPP & $313 \pm 16$ & $-57 \pm 17$ \\
TASPP & $282 \pm 30$ & $-119 \pm 28$ \\
TDASPP & $271 \pm 15$ & $-44 \pm 16$ \\
ZnTPP & $537 \pm 50$ & $513 \pm 29$ \\
HSq & $598 \pm 96$ & $682 \pm 92$ \\
HSq* & $597 \pm 118$ & $870 \pm 111$ \\
DCA & $605 \pm 106$ & $239 \pm 89$ \\
\hline
\end{tabular}

not in fact give such a significant difference and thus, the influence of the dodecyl groups is small (irrespective of the formal cavity size). The explanation is that $\Theta_{\mathrm{gr}}\left(\Theta_{\mathrm{ex}}-\Theta_{\mathrm{gr}}\right)$ should also rise to compensate the increase of cavity radii. Thus, using the cavity radii [38] and $\Xi$ parameters (in Table 3$)$, the product $\Theta_{\mathrm{gr}}\left(\Theta_{\mathrm{ex}}-\right.$ $\left.\Theta_{\mathrm{gr}}\right)$ is proportional to $1,4.6$ and 4.4 for TPP, TASPP and TDASPP, respectively.

\subsection{Spectral shifts of other centrosymmetric molecules correlated by quadrupole dielectric model}

As mentioned above, there are few reported cases in the literature which apply the dielectric dipolar models to study the absorption spectral shift of centrosymmetric molecules. In order to compare the results obtained from quadrupolar interaction in porphyrins studied here, we also applied the quadrupole dielectric model to some of these molecules like zinc tetraphenylporphyrin (ZnTPP) [14], squaraine (HSq) [15] and 9,10-dicyanoanthracene (DCA) [26]. Also, the model was applied to fluorescence emission shifts in squaraine $\left(\mathrm{HSq}^{*}\right)$ [15]. Fig. 3(b), (c) and (d) show the correlation in these molecules with the Eq. (15) and the results are in Table 3 . By contrast to the previous porphyrins, these molecules do not show good correlation only with $f\left(n^{2}\right)$. The correlations improve considerably when the BMO model is applied. However, when Eq. (15) is used, the correlations are in general better than those obtained with the BMO model since the quadrupolar model has one less parameter and all the average values are larger than the corresponding statistic errors. Also, contrasting to the previous porphyrins, in these three centrosymmetrical molecules the quadrupolar interaction terms are as important as the dispersion terms or even more important to describe the corresponding spectral shift in the range of solvents studied (Table 4). Also, the excited state quadrupole moments are larger than the ground state quadrupole moments for these molecules, 
indicating that there is an increase of quadrupole moment from the ground to the excited state, if the cavity radii are the same and the dipole moments are still equal to zero.

\section{Conclusions}

Using the classical dielectric models based on dipolar interactions, it was shown that dispersion forces dominate the solvatochromic effect in free base porphyrins studied. Interestingly, in ZnTPP, HSq and DCA, to which the solvatochromic effects were previously investigated, the quadrupolar interactions are more important. The application of dipolar dielectric models to centrosymmetric molecules affords good correlations of the spectral shifts, but incorrect interpretations can be extrapolated. Therefore, we believe that the quadrupolar dielectric model developed herein which includes the quadrupolar interaction between the solute quadrupole moment and polarized solvent molecules (represented by the dielectric continuum) is a more adequate description of the spectral shifts observed in centrosymmetric solute molecules. According to the model, the polarization terms caused by dipole and quadrupole moments are linearly correlated thus explaining why the BMO model shows good correlation with centrosymmetric solutes.

\section{Acknowledgements}

The Referee is acknowledged for several relevant and helpful comments of the manuscript. The authors are grateful to Dr. J.A.B. Ferreira for the useful discussions. This work was supported by CQE4/FCT and project POCTI/35398/QUI/2000 and 3rd Quadro Comunitário de Apoio (FEDER). D.M. Togashi thanks FCT for the award of BPD5739/2001.

\section{Appendix A}

The reaction field created by an axial quadrupole moment can be obtained using the dipole moment (Onsager description). To calculate the reaction field it is necessary to know the potential in the cavity due to the multipole itself as well as with the solvent molecules which are represented by the polarizable dielectric continuum. The potential function of an axial quadrupole can be represented by

$\phi=\frac{\Theta}{2 r^{3}}\left(3 \cos ^{2} \theta-1\right)$,

where $\theta$ is the angle between the axial symmetry and the vector $r$ is represented by the scalar form in the above equation where the origin is the center of sphere, of radius $a$, which represents the cavity. For the case of dielectric sphere of radius $a$ immersed in a dielectric medium $\varepsilon$, the outside and inside potential functions which satisfy Laplace's equation $(\Delta \phi=0)$ [27] are

$$
\begin{aligned}
& \phi_{\text {in }}=\sum_{i=0}^{\infty}\left(\alpha_{i}^{0} r^{i}+\frac{\beta_{i}^{0}}{r^{i+1}}\right) P_{i}(\cos \theta), \\
& \phi_{\text {out }}=\sum_{i=0}^{\infty}\left(\alpha_{i} r^{i}+\frac{\beta_{i}}{r^{i+1}}\right) P_{i}(\cos \theta) .
\end{aligned}
$$

The terms $P_{i}(\cos \theta)$ are the Legendre functions. The boundary conditions for the problem are: (a) the outside potential at infinite distance has to be zero, $\lim \phi_{\text {out }}=0$, (b) the potentials are equal on both sides of sphere, $\phi_{\text {in }}(r=a)=\phi_{\text {out }}(r=a)$ and (c) potential functions are continuous across both sides, $\varepsilon\left(\partial \phi_{\text {out }} / \partial r\right)_{r=a}=$ $\varepsilon_{\text {in }}\left(\partial \phi_{\text {in }} / \partial r\right)_{r=a}$. Using the boundary condition (a), the coefficients $\alpha_{i}$ are zero and the inside potential have to match the only source of field lines due to the axial quadrupole moment. Then, the potential functions are reduced to

$\phi_{\text {in }}=\sum_{i=0}^{\infty}\left[\alpha_{i}^{0} r^{i} P_{i}(\cos \theta)+\frac{\Theta}{2 r^{3}}\left(3 \cos ^{2} \theta-1\right)\right]$,

$\phi_{\text {out }}=\sum_{i=0}^{\infty} \frac{\beta_{i}}{r^{i+1}} P_{i}(\cos \theta)$.

Using the boundary conditions (b) and (c), the coefficients $\alpha_{i}^{0}$ and $\beta_{i}$, for $i=0$, are zero. However, for quadrupole moment $i=2$ :

$\alpha_{2}^{0} a^{2}+\frac{\Theta}{a^{3}}=\frac{\beta_{2}}{a^{3}}$,

$\varepsilon_{\text {in }}\left[2 \alpha_{2}^{0} a-\frac{3 \Theta}{a^{4}}\right]=\varepsilon\left[-\frac{3 \beta_{2}}{a^{4}}\right]$.

Solving the system, the potential functions for the axial quadrupole moment on the center of sphere of radius $a$ and inner dielectric $\varepsilon_{\text {in }}$ immersed in the dielectric medium $\varepsilon$ are

$$
\begin{aligned}
\phi_{\text {in }}= & -\frac{1}{a^{5}} \frac{3\left(\varepsilon-\varepsilon_{\text {in }}\right)}{3 \varepsilon+2 \varepsilon_{\text {in }}} r^{2} \frac{\Theta}{2}\left(3 \cos ^{2} \theta-1\right) \\
& +\frac{\Theta}{2 r^{3}}\left(3 \cos ^{2} \theta-1\right), \\
\phi_{\text {out }}= & \frac{5 \varepsilon_{\text {in }}}{3 \varepsilon+2 \varepsilon_{\text {in }}} \frac{\Theta}{2 r^{3}}\left(3 \cos ^{2} \theta-1\right) .
\end{aligned}
$$

According to Eq. (A.8), the inner sphere potential is the superposition of the axial quadrupole moment in the dielectric $\varepsilon_{\text {in }}=1$ (vacuum) and a reaction field defined as

$$
R^{(Q)}=\frac{\Theta}{a^{5}} \frac{3(\varepsilon-1)}{3 \varepsilon+2} .
$$




\section{References}

[1] N. Mataga, T. Kubota, Molecular Interaction and Electronic Spectra, Marcel Dekker, New York, 1970.

[2] A.T. Amos, B.L. Burrows, Adv. Quantum Chem. 7 (1973) 289.

[3] C.J. Cramer, D.G. Truhlar, Chem. Rev. 99 (1999) 2161.

[4] J. Tomasi, M. Persico, Chem. Rev. 94 (1994) 2027.

[5] P. Suppan, N. Ghoneim, Solvatochromism, Royal Society of Chemistry, Cambridge, UK, 1997.

[6] L. Onsager, J. Am. Chem. Soc. 58 (1936) 1486.

[7] N.S. Bayliss, J. Chem. Phys. 18 (1950) 292.

[8] Y. Ooshika, J. Phys. Soc. Jpn. 9 (1954) 594.

[9] E.G. McRae, J. Phys. Chem. 61 (1957) 562.

[10] I. Renge, Chem. Phys. Lett. 185 (1991) 231.

[11] R.J. Abraham, L. Cavalli, K.G.R. Pachler, Mol. Phys. 11 (1966) 471.

[12] L. Došen-Mićović, V. Žigman, J. Chem. Soc., Perkin Trans. II (1985) 625.

[13] Y. Taniguchi, H. Takaya, P.T.T. Wong, E. Whalley, J. Chem. Phys. 75 (1981) 4815.

[14] T.M.R. Viseu, G. Hungerford, A.F. Coelho, M.I.C. Ferreira, J. Phys. Chem. B 107 (2003) 13300.

[15] C.A.T. Laia, S.M.B. Costa, Phys. Chem. Chem. Phys. 1 (1999) 4409.

[16] A.S. Tatikolov, S.M.B. Costa, J. Photochem. Photobiol. A 140 (2001) 147.

[17] S.H. Gallagher, R.S. Armstrong, P.A. Lay, C.A. Reed, J. Phys. Chem. 99 (1995) 5817.

[18] J.L. Rivail, D. Rinaldi, Chem. Phys. 18 (1976) 233.

[19] K.V. Mikkelsen, A. Cesar, H. Ågren, A.J. Jøgen, J. Chem. Phys. 103 (1995) 9010.

[20] L. Došen-Mićović, D. Jeremić, N.L. Allinger, J. Am. Chem. Soc. 105 (1983) 1716.
[21] B. Strehmel, A.M. Sarker, H. Detert, Chemphyschem 4 (2003) 249.

[22] N. Ghoneim, P. Suppan, Spectrochim. Acta, Part A 51 (1995) 1043.

[23] M.A. Aguilar, J. Phys. Chem. A 105 (2001) 10393.

[24] H.J. Kim, J.T. Hynes, J. Chem. Phys. 93 (1990) 5194.

[25] H.J. Kim, J. Chem. Phys. 105 (1996) 6818.

[26] A.F. Olea, D.R. Worrall, F. Wilkinson, S.L. Williams, A.A. Abdel-Shafi, Phys. Chem. Chem. Phys. 4 (2002) 161.

[27] C.J.F. Böttcher, Theory of Electric Polarization, Elsevier, Amsterdam, 1973.

[28] M.D. Newton, H.L. Friedman, J. Chem. Phys. 88 (1988) 4460.

[29] D.R. Lide, Handbook of Chemistry and Physics, 72nd ed., CRC Press, Boca-Raton, Boston, 1991.

[30] S.L. Murov, I. Carmichael, G.L. Hug, Handbook of Photochemistry, second ed., Marcel Dekker, New York, 1993.

[31] A.J. Hudson, T. Richardson, J.P. Thirtle, G. Roberts, R.A.W. Johnstone, A.J.F.N. Sobral, Mol. Cryst. Liq. Cryst. 235 (1993) 103.

[32] I. Renge, U. Mölder, I. Koppel, Spectrochim. Acta, Part A 41 (1985) 967.

[33] U. Even, J. Magen, J. Jortner, J. Friedman, H. Levanon, J. Chem. Phys. 77 (1982) 4374.

[34] M. Meot-Ner, A.D. Adler, J. Am. Chem. Soc. 97 (1975) 5107.

[35] W.K. Plucknett, H.P. Woods, J. Phys. Chem. 67 (1963) 271.

[36] M. Rajeswaran, T.N. Blanton, N. Zumbulyadis, D.J. Giesen, C. Conesa-Moratilla, S.T. Misture, P.W. Stephens, A. Huq, J. Am. Chem. Soc. 124 (2002) 14450.

[37] A. Ghosh, J. Am. Chem. Soc. 117 (1995) 4691.

[38] The Onsager cavity was obtained from the molecular model ACD/ ChemSketch Version 5.12 (2002), Calculating the molar volumes. The radii of TPP, TASPP and TDASPP are $5.8 \AA, 6.2 \AA$ and 8.2 $\AA$, respectively. 